EPJ manuscript No.

(will be inserted by the editor)

\title{
Understanding nucleon structure using lattice simulations
}

\section{Recent progress on three different structural observables}

\author{
Wolfram Schroers \\ John von Neumann-Institut für Computing NIC/DESY, 15738 Zeuthen, Germany \\ Received: date / Revised version: date
}

\begin{abstract}
This review focuses on the discussion of three key results of nucleon structure calculations on the lattice. These three results are the quark contribution to the nucleon spin, $J_{q}$, the nucleon- $\Delta$ transition form factors, and the nucleon axial coupling, $g_{A}$. The importance for phenomenology and experiment is discussed and the requirements for future simulations are pointed out.

Preprint number: DESY 06-194
\end{abstract}

PACS. 12.38.Gc Lattice gauge theory, nucleon structure

\section{Introduction}

In recent years lattice gauge theory has become a mature and reliable way to investigate the structure of strong interactions. It provides a model-independent way to do calculations in QCD. However, contemporary lattice computations become extremely costly at quark masses corresponding to pion masses below $500 \mathrm{MeV}$. Nature, however, has chosen the pion mass to be only $140 \mathrm{MeV}$. The lightness of the pseudoscalar mesons is due to the mechanism of spontaneous chiral symmetry breaking. If, however, we can investigate only the regime of heavy quarks, where chiral symmetry is broken explicitly by the quark mass, we might not describe physics accurately at light quark masses.

To address and overcome this challenge, three different procedures have been proposed and are actively pursued: (i) Pushing existing simulations with Wilson-type quarks down to smaller quark masses by relying on improved algorithms and faster computers [1, (ii) using a hybrid action approach by using different formulations for sea- and valence-quarks [2, and (iii) doing simulations using dynamical Ginsparg-Wilson formulations, such as DomainWall fermions 3 or Overlap fermions [4. The last approach is certainly the most challenging and demanding one since the entire parameter space has to be explored again. This applies also to heavy quarks, a regime in which Ginsparg-Wilson fermions are about 30 to 100 times more expensive than standard Wilson-type fermions.

The hybrid action ansatz is an excellent compromise between quark mass and performance, but suffers from conceptual problems. First of all, the hybrid theory breaks unitarity at finite lattice spacing. Thus, it cannot act as an effective theory at finite lattice spacing, and the existence of the continuum limit is crucial. Furthermore, usually staggered-type quarks are being used for the sea with the square-root being taken of the determinant. It is not clear if the procedure of taking the square root commutes with taking the continuum limit, see e.g. 5] for a recent review. Finally, the matching of sea- and valence-quark masses is prescription-dependent, and particular choices may give rise to additional possibly large $\mathcal{O}\left(a^{2}\right)$ artifacts [6].

In this review we focus on three observables with relevance to phenomenological and experimental applications. The first one is the quark contribution to the nucleon spin, $J_{q}$. The second one is the transition form factors of the nucleon- $\Delta$ transition. The third one is the nucleon axial coupling, $g_{A}$. The former two of these quantities have so far been understood qualitatively, but a precise matching between the light quark regime and the lattice - possibly by chiral perturbation theory or an effective model of the strong interaction like [7] - still remains to be done. For the latter observable it has been shown that lattice data can in fact be consistent with experiment when fitting it using the leading logarithmic chiral perturbation theory expression. This achievement marks a milestone in the field of nucleon structure.

\section{Quark contribution to nucleon spin}

The quark contribution to the spin of the nucleon has been under intense scrutiny after the observation that only about $(20 \pm 15) \%$ of the nucleon spin arises from the quark spin [8]. Recently, it has been realized how the use of GPDs 9, 11, 10, provides the means to directly compute 
Wolfram Schroers: Understanding nucleon structure using lattice simulations

the quark contribution to the nucleon spin via the energy momentum tensor [10]

$$
J_{q}=\lim _{t \rightarrow 0}\left(A_{20}^{\mathrm{u}+\mathrm{d}}(t)+B_{20}^{\mathrm{u}+\mathrm{d}}(t)\right) .
$$

The virtuality $t$ is given by $t \equiv\left(p^{\prime}-p\right)^{2}$, where $p^{\prime}$ and $p$ are the nucleon's incoming and outgoing momenta. The generalized form factors, $A_{20}^{\mathrm{u}+\mathrm{d}}(t)$ and $B_{20}^{\mathrm{u}+\mathrm{d}}(t)$, show up in the parameterization of the nucleon's energy-momentum tensor. For further details and the exact definition consult [10]. The challenge is to understand which fraction of the nucleon spin, $J_{N}=1 / 2$, arises from the quark spin, $1 / 2 \Sigma_{q}$, the quark orbital angular momentum, $L_{q}$, and which fraction comes from gluon contributions, $J_{g}$ :

$$
J_{N}=1 / 2=J_{q}+J_{g}=1 / 2 \Sigma_{q}+L_{q}+J_{g} .
$$

The value of $\Sigma_{q}$ has been known before 12 . The new ingredient is the ability to directly calculate $J_{q}$, and thus also $L_{q}$. To this end, there is no experimental determination of that quantity. The first computation of $J_{q}$ on the lattice has been done in [12. This calculation only utilizes quenched Wilson fermions, but features a calculation of the disconnected contribution using noisy estimators. A later calculation [13. calculates all generalized form factors in the energy-momentum tensor separately and at the same time a publication [14 features full QCD and introduces an improved technology to extract form factors from matrix elements. Higher moments of GPDs have also been computed [15].

As of today, the understanding gained from the world of pions weighing $500 \mathrm{MeV}$ and beyond is that the quark contribution to the nucleon spin is about $70 \%$, all of which comes from the quark spin alone. The remaining $30 \%$ comes from the gluons. The quark orbital angular momentum is negligibly small due to a cancellation between the contributions of $\mathrm{u}$ - and d-quarks [2].

This result differs from the finding outlined above which indicates that this quantity can be expected to substantially depend on the pion mass. The cancellation of the orbital angular momentum for $\mathrm{u}$ - and d-quarks is an interesting qualitative feature. The insight that the nucleon in the heavy pion world receives a larger fraction of its spin from quarks rather than gluons is compatible with expectations from the non-relativistic quark model, but the exact interpolation between the heavy quark and the light quark regime can give further insight into how the strong interaction operates.

The extrapolation to the chiral regime, however, has not yet been possible and hence a precise quantitative matching with Nature has not yet been established. Although Ref. 16] suggests a rather flat expression it is yet unclear whether the same straight line is to be used for the light quark regime as the one fitting the simulations. In this situation it is inevitable to perform similar calculations at smaller pion masses before a matching between lattice and small-scale expansion schemes can be established and a definitive prediction from the lattice can be provided.

Further investigations from several groups are underway and all three different paths outlined in Sec. 1 are taken to resolve this important question. We can conclude, however, that the technology and understanding of how to compute these matrix elements are available and can be deployed easily once sufficiently light pion masses are available.

\section{$3 N \rightarrow \Delta$ transition form factors}

A key question is whether the baryon states of QCD are spherical or deformed. Although the nucleon is easily accessible in exclusive and inclusive scattering experiments, it cannot have a spectroscopic quadrupole moment since it has $\operatorname{spin} J_{N}=1 / 2$. The excited states with spin $3 / 2$ and above can have a quadrupole moment, but these are not easily accessible in experiments. The only way to learn about deformations of the low-lying baryon spectrum is to consider transitions between the nucleon and the first excited state, the $\Delta(1232)$ resonance. Experimentally, a flurry of activity has recently lead to several important and exciting results [17].

The nucleon- $\Delta$ transition can be parameterized using three form factors - the dominant magnetic dipole form factor, $\mathcal{G}_{M 1}$, the electric quadrupole, $\mathcal{G}_{E 2}$, and the Coulomb quadrupole, $\mathcal{G}_{C 2}$. Should the nucleon- $\Delta$ system be deformed, the latter two form factors will not vanish. Should the system be spherical, only the magnetic dipole form factor will be non-zero.

On the lattice, publications reporting the successful computation of these transition form factors are [18. This set of calculations used unquenched Wilson fermions with pion masses beyond $600 \mathrm{MeV}$ and quenched Wilson fermions with pion masses larger than $370 \mathrm{MeV}$. Later it has been attempted to apply these techniques also for hybrid actions [19], but to this end the statistical error bars on the quadrupole form factors turn out to be too large.

From these studies it has been clearly established, that the nucleon- $\Delta$ system is indeed deformed. The sign and the order of magnitude of the quadrupole form factors $\mathcal{G}_{E 2}$ and $\mathcal{G}_{C 2}$ was extracted successfully. The heavy pion world in fact is similar to Nature for these observables.

However, the extrapolation to the physical pion masses yields an inconsistency for $\mathcal{G}_{C 2}$ at values below $Q^{2}<$ $0.2 \mathrm{GeV}^{2}$. This discrepancy has been addressed recently in the framework of chiral perturbation theory [20]. It appears plausible that the discrepancy in fact arises from the inadequacy of a linear chiral extrapolation - it still remains to be seen if lattice data at smaller pion masses can indeed verify the pion mass dependence suggested in [20].

\section{Nucleon axial coupling $g_{A}$}

The investigation of the nucleon axial coupling has a long history on the lattice, see 21] for recent reviews. Several groups have performed investigations using a wide array of different lattice actions, spacings, volumes, and pion masses.

Recently, two independent papers [22] and 23] have appeared showing how current lattice data can in fact 


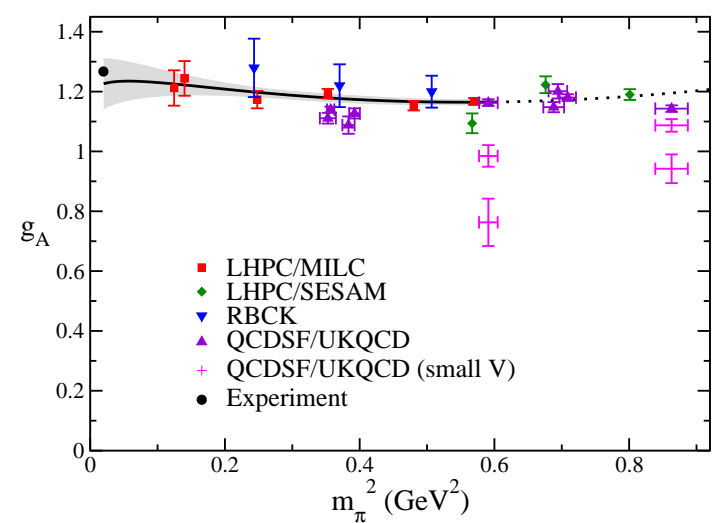

Fig. 1. Full QCD computations of the nucleon axial coupling, $g_{A}$. The line shows the fit of the leading logarithmic $\chi \mathrm{PT}$ expression to the hybrid lattice data from the LHPC collaboration. Results from other groups are plotted, but not included in the fit. Figure taken from Ref. 22, QCDSF data updated from Ref. 23.

be combined with chiral perturbation theory to arrive at the experimental value. Figure 4 shows the application of the leading logarithmic expression from $\chi \mathrm{PT}$ to the hybrid data computed by the LHPC collaboration in 22 . The gray-shaded error band shows the error arising from statistical uncertainties only. The fit yields quantitative agreement with the experimental value.

However, the applicability of the leading order chiral perturbation theory expression to the pion masses available has been questioned in 24. The flat behavior at pion masses beyond $300 \mathrm{MeV}$ is attributed to fine-tuning between different terms in the expansion. On the other hand, the expansion can still be consistent with experiment when applied to lattice calculations employing pion masses as large as $600 \mathrm{MeV}$ 23. It is perhaps fair to say that the exact range of applicability of $\chi \mathrm{PT}$ is under debate. Nonetheless, the striking agreement between the fit of lattice data and the experimental value mark an important milestone for the lattice treatment of nucleon structure.

\section{Summary}

We have given three examples of recent lattice calculations which are of great interest to both phenomenologists and experimentalists alike. The limiting factor of all these lattice results, however, is their limitation to rather large quark masses. Currently, the question of chiral extrapolations is under debate and the applicability depends strongly on the observable. While some groups successfully apply fits to pion masses as large as $600 \mathrm{MeV}$, other groups believe that pion masses lower than $300 \mathrm{MeV}$ are essential. While the latter mass regime has not been reachable so far, we believe that the upcoming generation of lattice calculations will be able to settle the debate.

This work was supported in part by the DFG, contract FOR 465 (FG Gitter-Hadronen-Phänomenologie), and in part by the EU Integrated Infrastructure Initiative Hadron Physics (I3HP), contract number RII3-CT-2004506078 .

\section{References}

1. K. Jansen et al., these proceedings; M. Göckeler et al., arXiv:hep-lat/0610066

2. J. W. Negele et al., Nucl. Phys. Proc. Suppl. 128 (2004) 170; D. B. Renner et al. [LHP Collaboration], Nucl. Phys. Proc. Suppl. 140 (2005) 255; Ph. Hägler, J. W. Negele, D. B. Renner, W. Schroers, T. Lippert and K. Schilling [LHPC Collaboration], Eur. Phys. J. A 24S1 (2005) 29; R. G. Edwards et al. [LHPC Collaboration], PoS LAT2005 (2006) 056; R. G. Edwards et al., arXiv:hep-lat/0610007

3. R. Tweedie et al. [UKQCD and RBC Collaborations], PoS LAT2005 (2006) 096.

4. N. Cundy, S. Krieg, A. Frommer, T. Lippert and K. Schilling, Nucl. Phys. Proc. Suppl. 140, 841 (2005); N. Cundy, S. Krieg and T. Lippert, PoS LAT2005, 107 (2006); S. Schäfer, arXiv:hep-lat/0609063.

5. S. Dür, PoS LAT2005, 021 (2006).

6. O. Bär, C. Bernard, G. Rupak and N. Shoresh, Phys. Rev. D 72, 054502 (2005).

7. K. Goeke, J. Ossmann, P. Schweitzer and A. Silva, Eur. Phys. J. A 27, 77 (2006).

8. R. D. Ball, S. Forte and G. Ridolfi, Phys. Lett. B 378 (1996) 255 .

9. D. Müller, D. Robaschik, B. Geyer, F. M. Dittes and J. Horejsi, Fortsch. Phys. 42 (1994) 101.

10. X. D. Ji, Phys. Rev. Lett. 78 (1997) 610.

11. A. V. Radyushkin, Phys. Rev. D 56 (1997) 5524.

12. N. Mathur, S. J. Dong, K. F. Liu, L. Mankiewicz and N. C. Mukhopadhyay, Phys. Rev. D 62 (2000) 114504.

13. M. Göckeler, R. Horsley, D. Pleiter, P. E. L. Rakow, A. Schäfer, G. Schierholz and W. Schroers [QCDSF Collaboration], Phys. Rev. Lett. 92 (2004) 042002.

14. P. Hägler, J. Negele, D. B. Renner, W. Schroers, T. Lippert and K. Schilling [LHPC collaboration], Phys. Rev. D 68, 034505 (2003).

15. P. Hägler, J. W. Negele, D. B. Renner, W. Schroers, T. Lippert and K. Schilling [LHPC Collaboration], Phys. Rev. Lett. 93 (2004) 112001.

16. J. W. Chen and X. d. Ji, Phys. Rev. Lett. 88 (2002) 052003.

17. G. Blanpied et al. [LEGS Collaboration], Phys. Rev. Lett. 76, 1023 (1996); R. Beck et al., Phys. Rev. C 61, 035204 (2000); C. Mertz et al., Phys. Rev. Lett. 86, 2963 (2001); N. F. Sparveris et al. [OOPS Collaboration], Phys. Rev. Lett. 94, 022003 (2005); K. Joo et al. [CLAS Collaboration], Phys. Rev. Lett. 88, 122001 (2002); A. M. Bernstein, Eur. Phys. J. A 17, 349 (2003).

18. C. Alexandrou et al., Phys. Rev. D 69, 114506 (2004); C. Alexandrou, Ph. de Forcrand, H. Neff, J. W. Negele, W. Schroers and A. Tsapalis, Phys. Rev. Lett. 94, 021601 (2005). 
19. C. Alexandrou et al., PoS LAT2005, 091 (2006).

20. V. Pascalutsa and M. Vanderhaeghen, Phys. Rev. Lett. 95 (2005) 232001.

21. W. Schroers, Nucl. Phys. A 755 (2005) 333; W. Schroers, Nucl. Phys. Proc. Suppl. 153 (2006) 277.

22. R. G. Edwards et al. [LHPC Collaboration], Phys. Rev. Lett. 96 (2006) 052001.

23. A. Ali Khan et al., arXiv:hep-lat/0603028.

24. V. Bernard and U. G. Meissner, Phys. Lett. B 639, 278 (2006). 